\title{
Understanding selectivity in solute-solute separation: definitions, measurements, and comparability
}

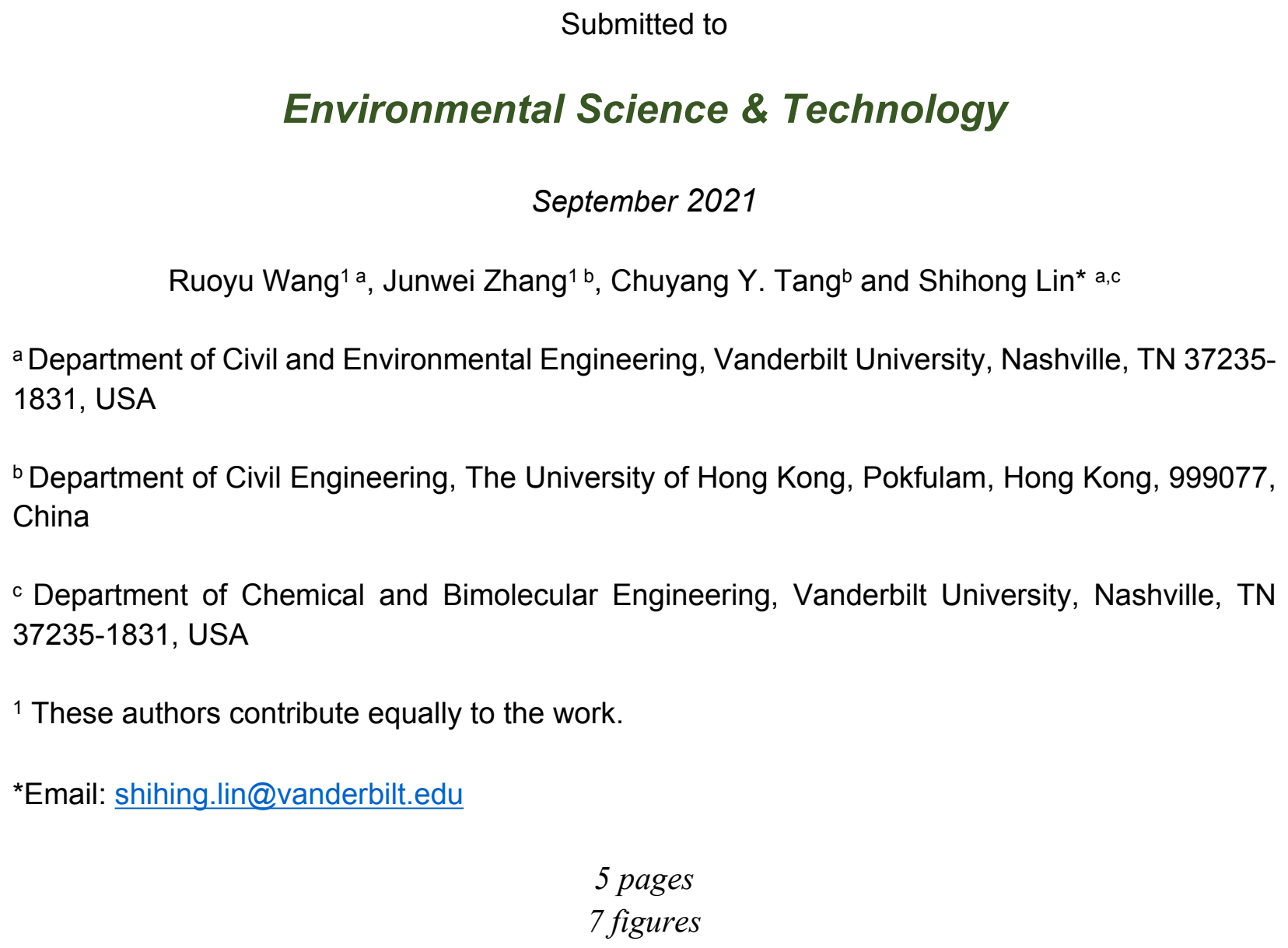


33

34

35

36
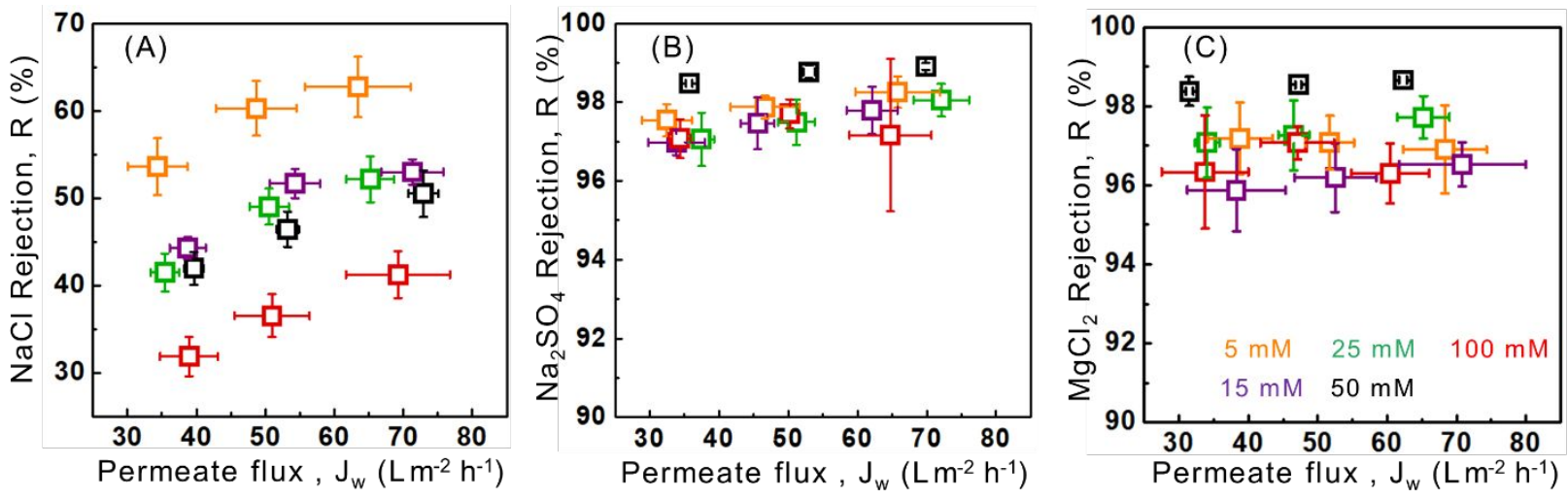

Figure S1. (A) $\mathrm{NaCl}$ rejection, (B) $\mathrm{Na}_{2} \mathrm{SO}_{4}$ rejection, and (C) $\mathrm{MgCl}_{2}$ rejection as a function of permeate flux under different feed concentrations.
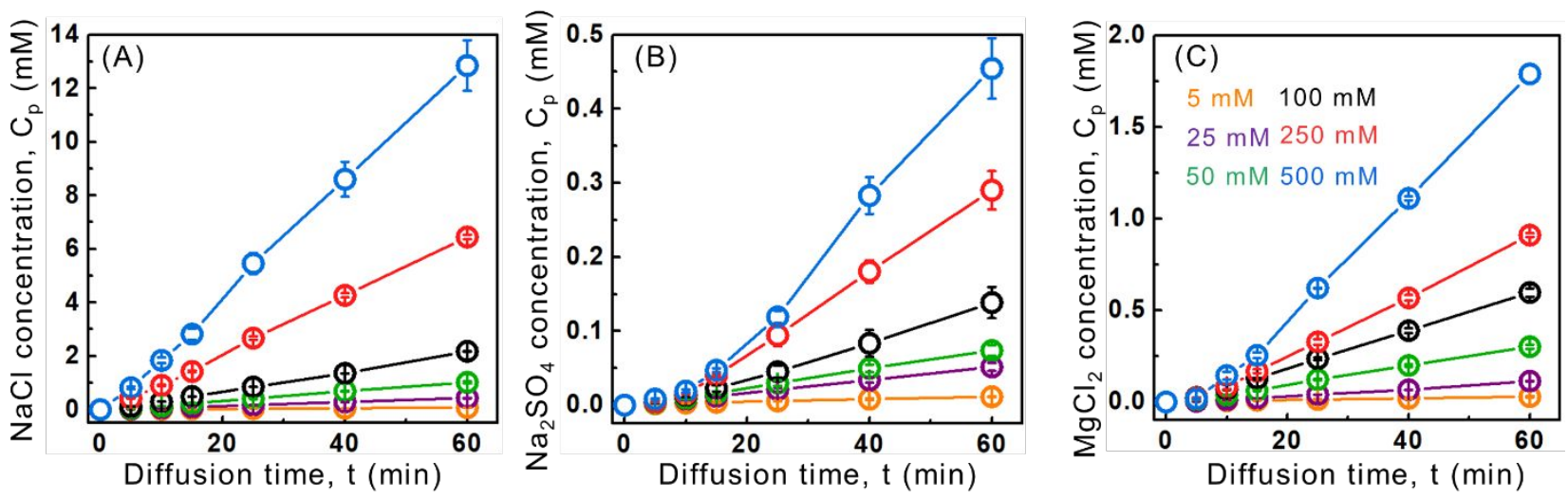

Figure S2. (A) NaCl, (B) $\mathrm{Na}_{2} \mathrm{SO}_{4}$ and (C) $\mathrm{MgCl}_{2}$ concentration in the receiving solution as a function of diffusion time under different feed concentrations. Conductivity of the receiving solution was measured over time and converted to permeate salt concentration. Osmosis was offset by the addition of sucrose in the receiving solution. 

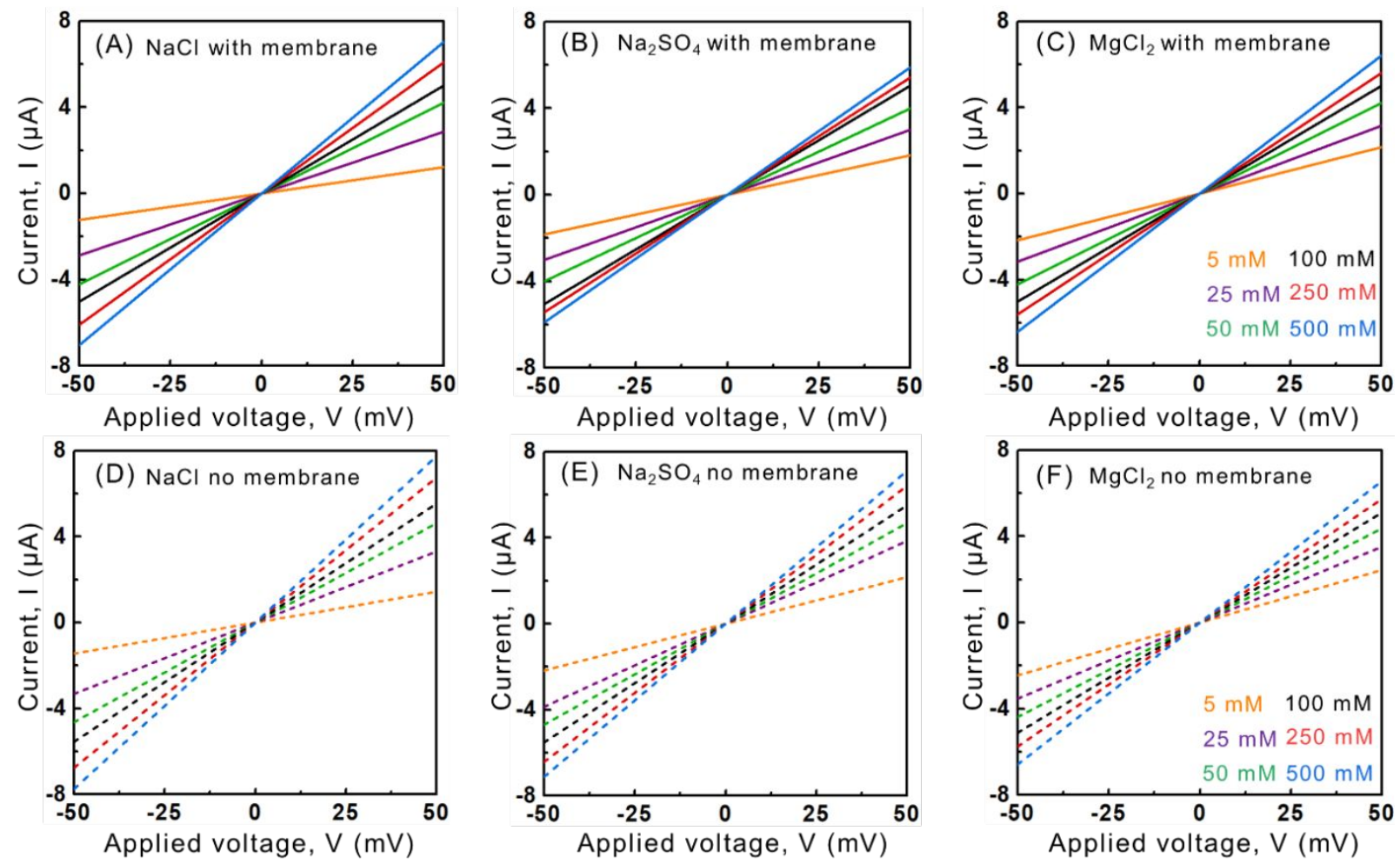

43

44

45

46

Figure S3. I-V curves with (A, B, C) and without membranes (D, E, F) under different feed concentrations. Linear sweep voltammetry was characterized within -50 to $50 \mathrm{mV}$ at a scan rate of $2 \mathrm{mV} / \mathrm{s}$.

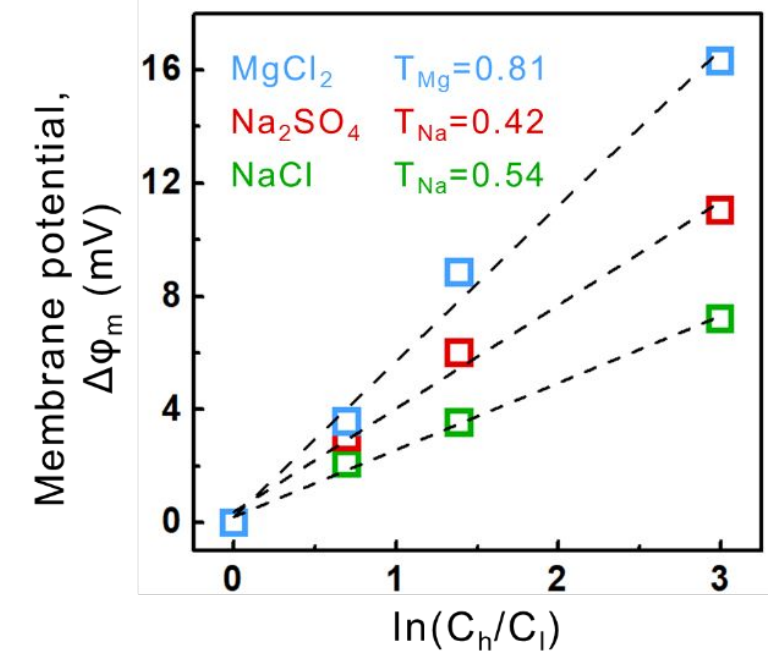

47

Figure S4. Dependence of membrane potential on the concentration ratio between the two chambers. The 

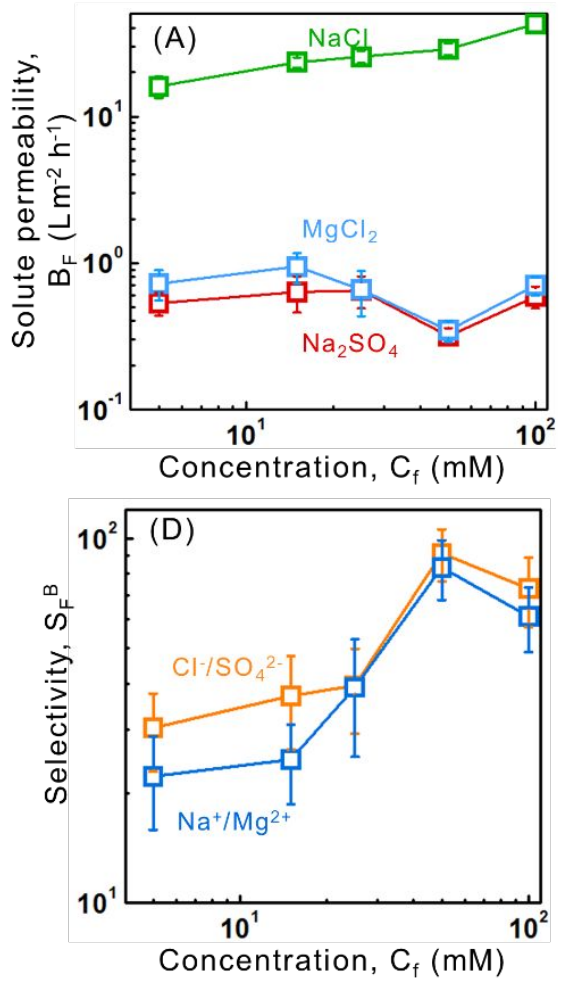
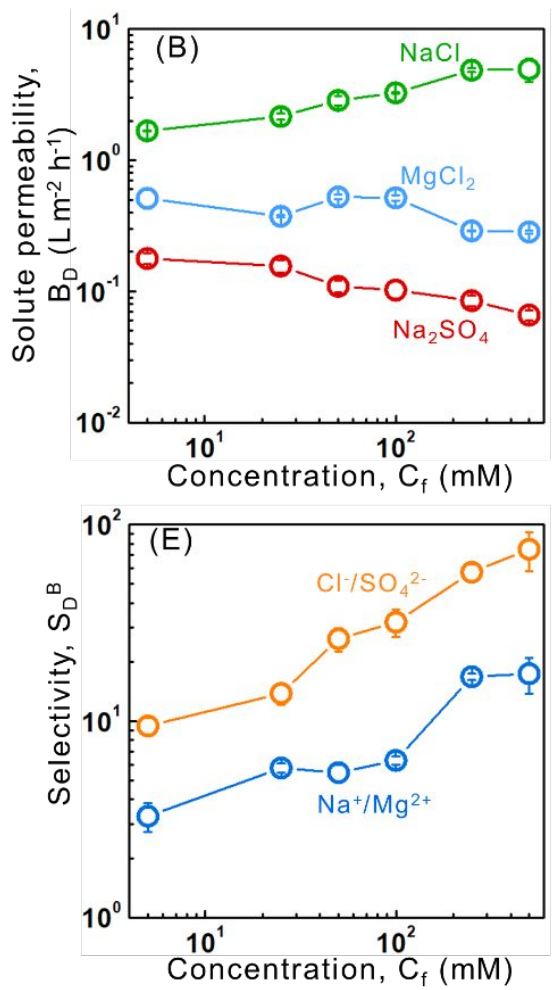
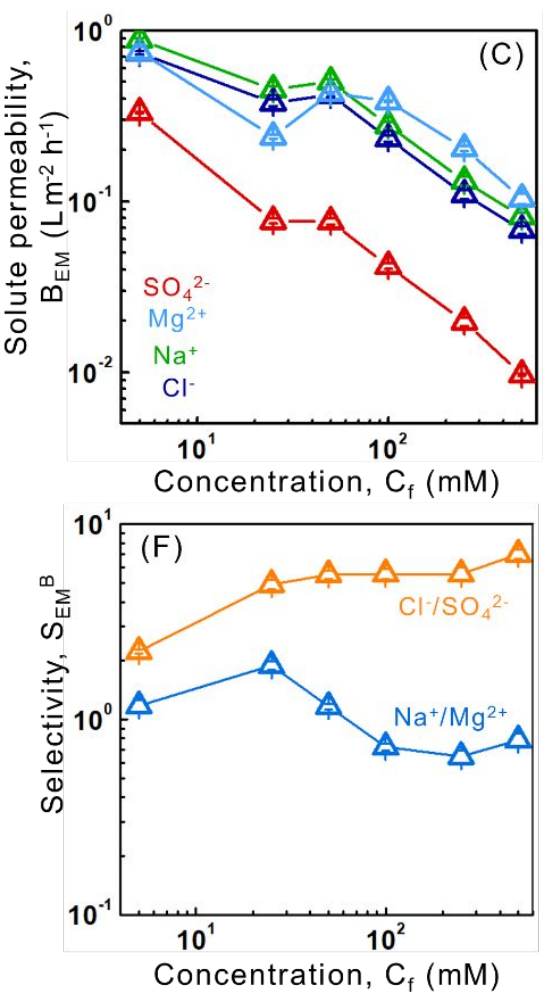

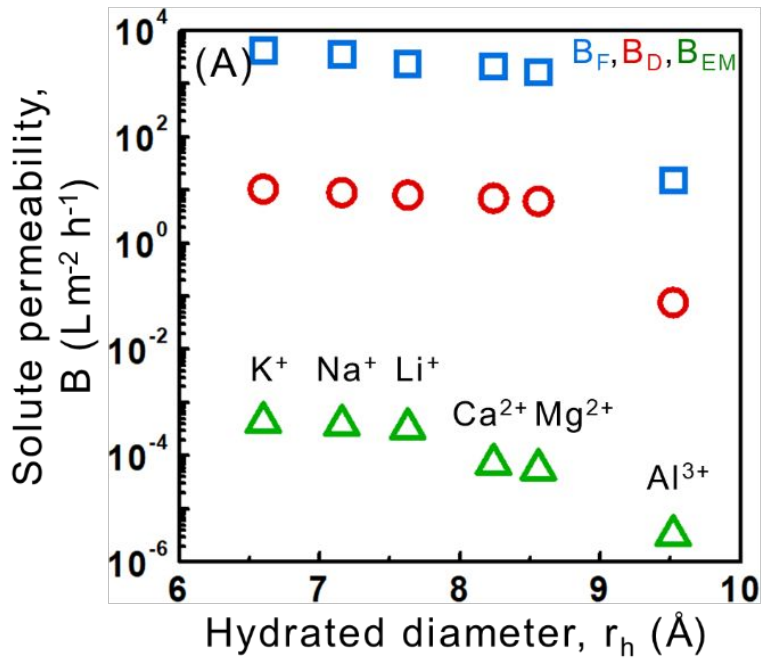

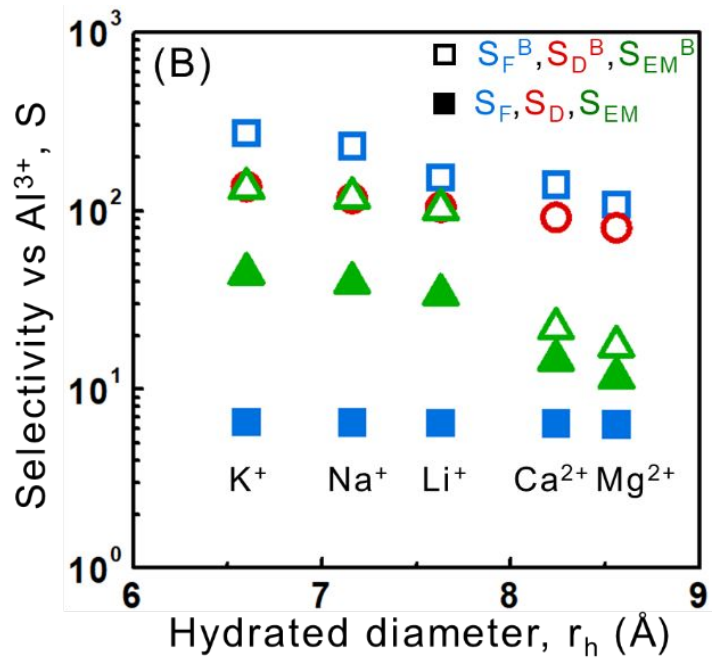

Figure S5. Solute permeability (A, B, C) and solute-solute selectivity (D, E, F) as a function of concentration calculated from filtration method, diffusion method and conductance method, respectively.

Figure S6. (A) Solute permeability and (B) $\mathrm{M}^{\mathrm{x}+} / \mathrm{Al}^{3+}$ selectivity of different definitions in a polycarbazoletype conjugated microporous polymer membrane. Permeability and selectivity were calculated based on original rejection, diffusion flux, and conductance data reported in reference. Concentration polarization effect was not considered. 

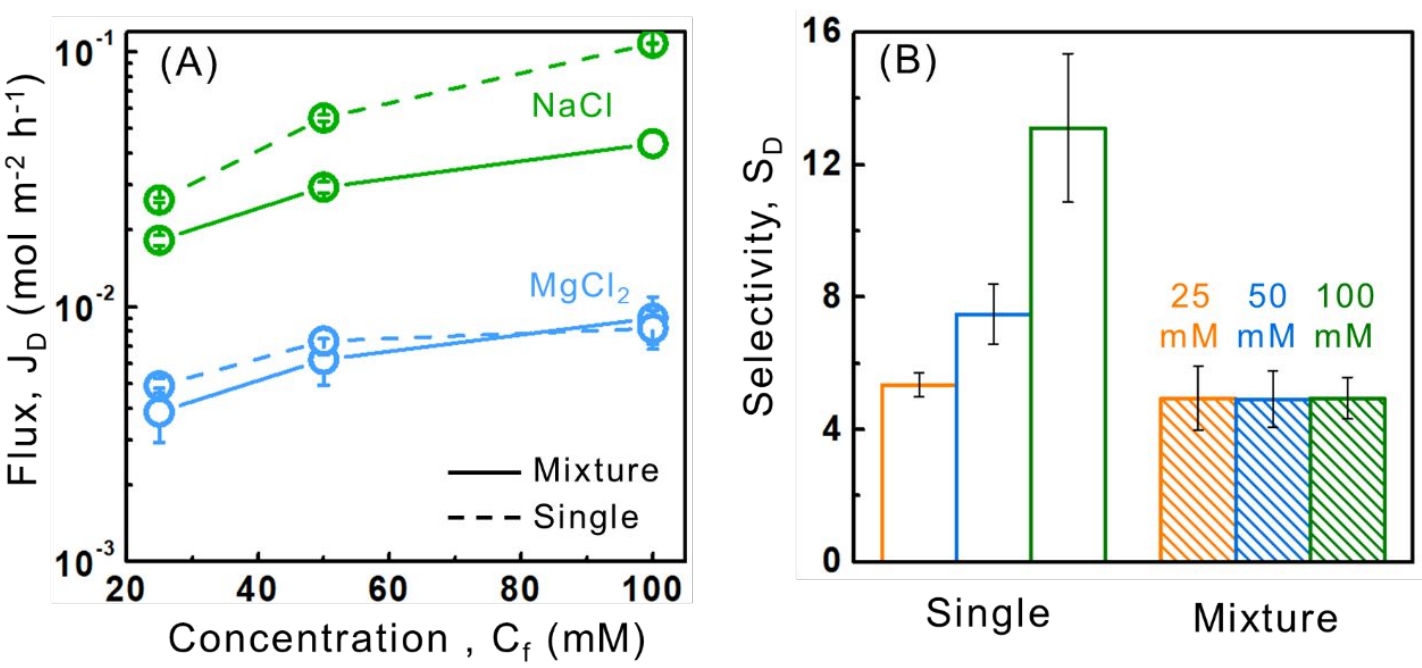

61

62 Figure S7. (A) Diffusion flux of $\mathrm{NaCl}$ and $\mathrm{MgCl}_{2}$ measured in single (dash) and mixture (solid) salt solutions.

63 Osmosis was not offset. (D) Comparison of $\mathrm{Na}^{+} / \mathrm{Mg}^{2+}$ selectivity defined on diffusion flux in single and

64 mixture salt solutions. Results under three feed concentrations (i.e., 25 mM, 50 mM, 100 mM for each salt)

65 were presented. 